\title{
Mehr Transparenz und Sicherheit für Eltern und Träger
}

\section{Die Finanzierung von Kindertageseinrichtungen - reformbedürftig}

Doris Beneke

Doris Beneke ist Referentin im Arbeitsfeld Tageseinrichtungen für Kinder im Diakonischen Werk der EKD in Berlin.

E-Mail beneke@diakonie.de
Die Finanzierung von Kindertageseinrichtungen ist in Deutschland höchst unterschiedlich geregelt. Das gesamte System zeichnet sich durch chronische Unterfinanzierung aus und wird den Anforderungen an eine qualitativ hochwertige Betreuung, Bildung und Erziehung von Kindern nicht gerecht.

Die Finanzierung von Kindertageseinrichtungen wird in Deutschland in insgesamt 16 Finanzierungssystemen geregelt. Grund dieser unterschiedlichen Regelungen ist der im Kinder- und Jugendhilfegesetz festgelegte Landesrechtsvorbehalt zur Finanzierung von Tageseinrichtungen für Kinder ( $\$ 74$ a SGB VIII). Damit ist es den Ländern möglich, völlig eigenständig die Finanzierung in Abweichung von den sonst im Kinder- und Jugendhilfegesetz vorgesehenen Finanzierungsformen in der Jugendhilfe zu regeln. Dies hat zu einer bunten Mischung unterschiedlichster Systeme geführt, die getrost als föderaler Flickenteppich bezeichnet werden kann.

\section{Wer finanziert eigentlich die Kindertageseinrichtungen?}

In fast allen Bundesländern sind die Länder, Kommunen, Träger und Eltern an der Finanzierung der Betriebskosten beteiligt. Aktuell ist ein Trend zur Kommunalisierung zu beobachten: Die Länder ziehen sich ganz oder teilweise aus der Finanzierung heraus und übertragen die Verantwortung auf die Kommunen. Für diese Verlagerung mag sprechen, dass die Kommunen im Idealfall die Mittel zielgenau und bedarfsgerecht einsetzen können. Andererseits sorgt eine geregelte Landesfinanzierung für eine einheitliche Ausstattung der Kindertageseinrichtungen in einem Bundesland und damit für vergleichbare Standards.

Die Träger bringen in unterschiedlicher Höhe entweder landesgesetzlich normierte Eigenanteile in zum Teil erheblicher
Höhe auf oder müssen die Differenz zu den nicht refinanzierten Kosten aufbringen. Diese entstehen, weil entweder bestimmte Betriebskosten - beispielsweise Verwaltungsausgaben - nicht anerkannt werden oder bei subjektorientierten Finanzierungsformen die tatsächlichen Betriebskosten über den vom öffentlichen Träger festgelegten und bewilligten Festbeträgen liegen. Dies ist insbesondere dann der Fall, wenn die tatsächlichen Personalkosten durch die Altersstruktur der Mitarbeitenden oder abweichende tarifliche Regelungen über den vom öffentlichen Träger festgelegten Kosten liegen. Im ungünstigsten Fall verbleibt bei einem freien Träger auch bei gesetzlich normierter Regelung ein Differenzbetrag nicht anerkannter Sach- oder Personalkosten, die seinen Eigenanteil zusätzlich erhöhen. In einigen Bundesländern werden auch vom Träger eingebrachte Eigenleistungen oder Elternleistungen als Eigenanteil bei der Berechnung der Zuschüsse anerkannt. Der Landes- oder Kommunalzuschuss wird in einigen Bundesländern als »Defizitdeckung « bezeichnet, obwohl dieser Begriff irreführend ist: Es geht nicht um den Ausgleich defizitärer Bewirtschaftung einer Einrichtung durch den Träger, sondern um die Berechnung des gesetzlich vorgesehenen Zuschussanteils des öffentlichen Trägers.

Eltern sind in der Regel an der Finanzierung der Kindertageseinrichtungen durch die Zahlung von Elternbeiträgen beteiligt. Einige Bundesländer wie Saarland, Rheinland Pfalz und Berlin haben Beitragsfreiheit für das letzte Kindergartenjahr eingeführt, teilweise sind weitere Beitragsfreistellungen politisch geplant. Die Erhebung von Elternbeiträgen erfolgt auf der Basis des $\mathbb{S} 90$ SGB VIII, der für die Inanspruchnahme von Kindertageseinrichtungen Teilnahmebeiträge vorsieht und den Ländern ermöglicht, Staffelungen der Beiträge nach Einkommensgruppen, Kinderzahl oder Zahl der Familienangehörigen vorzunehmen. Der Beitrag 
kann ganz oder teilweise erlassen werden oder vom öffentlichen Träger der Jugendhilfe übernommen werden, wenn die Belastung dem Kind oder seinen Eltern nicht zuzumuten ist.

In der Praxis gibt es mehr als nur 16 Länderregelungen und Staffelungssysteme, da nur ein Teil der Bundesländer, wie zum Beispiel Hamburg, Rheinland Pfalz und Sachsen, die Elternbeitragshöhe regelt. Etliche Länder haben die Festlegung der Beiträge auf die Kommune übertragen, jede Kommune entscheidet dann selbst über die Beitragshöhe. Das führt dazu, dass in einem Bundesland regional unterschiedliche Beiträge je nach Beschlusslage der Kommune zu entrichten sind. Wie teuer oder preiswert ein Platz für Eltern wird, hängt also vom Wohnort ab. Noch unübersichtlicher wird es, wenn die Beitragsfestlegung auch durch den Träger erfolgen kann, dann gibt es unter Umständen sogar in einer Kommune trägerspezifisch unterschiedlich hohe Beiträ- lich wurde in einer Bund Länder Vereinbarung die Einrichtung eines Sondervermögen über 2,15 Milliarden Euro zur Investitionsförderung beschlossen. Darüberhinaus wird sich der Bund im Rahmen der Umsatzsteuerverteilung an den Betriebskosten beteiligen.

\section{Objektfinanzierung versus Subjektfinanzierung}

Grob lassen sich zwei Grundstrukturen in den Finanzierungssystemen unterscheiden, die objektbezogene und die subjektbezogene Finanzierung.

- Bei der Objektfinanzierung erhält der Träger eine Zuwendung, in der Regel werden die in der Betriebserlaubnis anerkannten Gruppen und die dafür anerkannten Personalkosten bezuschusst. Kritiker bemängeln an dieser Form der Finanzierung die pauschale Bezuschussung des Trägers und der Einrichtung

\section{"Subjektfinanzierung verlangt Bedarfsfeststellung «}

ge. Die Höhe der Beiträge zeichnet sich durch eine breite Varianz aus, die sich auch noch nach Art und zeitlichem Betreuungsumfang unterscheidet. Von vergleichbaren Beitragshöhen kann in Deutschland nicht gesprochen werden. Eltern müssen bei einem Umzug mit erheblichen Kostenunterschieden für die Betreuung ihrer Kinder rechnen.

Das ist die Ausgangslage, vor der über eine Beteiligung des Bundes an der Finanzierung diskutiert wird. Und in dieser Situation erwartet die Konferenz der Jugend- und Familienminister allen Ernstes, dass die Verteilung der Bundesmittel so gestaltet wird, dass diese mit den Verfahren und Strukturen in den Ländern kompatibel ist. Die Umsetzung dieser Vorgabe dürfte mit riesigem Verwaltungsaufwand verbunden sein - Mittel, die dringend für den tatsächlichen Ausbau benötigt werden und nicht in einen zwangsläufig entstehenden bürokratischen Wasserkopf gehen dürfen. Bereits entwickelte Modelle wie die Einführung eines Geldleistungsgesetzes (»Kitageld «) werden bedauerlicherweise von den Akteuren nicht diskutiert. (1) Zwischenzeit- unabhängig von der Belegung. Sie vermissen Anreize zu Wettbewerb und wirtschaftlichem Handeln da auch nicht belegte Plätze und mögliche freie Personalressourcen bezuschusst werden. Jugendhilferechtlich wird diese Form der Finanzierung kritisiert, weil sie die Einrichtung finanziert und nicht die Kinder als Subjekte der Förderung. Dies müsste zumindest bei den Rechtsanspruchsplätzen gewährleistet werden, deren Finanzierung auf der Basis des jugendhilferechtlichen Dreiecksverhältnisses zu gestalten ist. Dementsprechend wäre die Entgeltfinanzierung die korrekte rechtliche Finanzierungsabwicklung . Damit verbunden wird die Forderung nach einer Neugestaltung der Finanzierung auf der Grundlage einer Subjektfinanzierung. (2)

- Subjektbezogene Finanzierung legt entweder die Auslastung des Angebotes zugrunde oder gibt den Leistungsberechtigten Mittel in die Hand, mit denen sie das Angebot bezahlen können(Gutschein). Innerhalb der Subjektfinanzierung haben sich in den Ländern unterschiedliche Formen entwickelt:
Pro belegtem Platz (Brandenburg, Mecklenburg, Thüringen), Finanzierung durch einen Gutschein (Hamburg, Berlin) oder Mischformen wie das bayerische Modell der markt- und qualitätsorientierten Steuerung. Hier wird die Förderung in Abhängigkeit von der Nutzungszeit gekoppelt mit einem aufwandsabhängigen Gewichtungsfaktor für Kinder mit Behinderungen, ausländische Kinder und Kinder unter drei Jahren.

Für die subjektbezogenen Finanzierungsformen lassen sich gravierende Mängel feststellen, die dringend zu einer Überarbeitung der Systeme führen müssten (3):

- Die starren Formen der Bedarfsfestlegung durch den öffentlichen Träger, die sich einseitig an Erwerbstätigkeit orientieren, verhindern einzelfallbezogene, an den Bedarfen der Kinder ausgerichtete sozialpädagogische Entscheidungen über den anzuerkennenden Betreuungsplatz und den Umfang der anerkannten Kosten. So geschehen in der Anfangsphase in Hamburg, wo die Einführung des Gutscheins durch Budgetierung der Mittel dazu führte, das nicht einmal alle berechtigten Empfänger eines Gutscheins bedient werden konnten.

- Der Zugang zu Gutscheinen und Buchungszeiten erfordert von Eltern die Fähigkeit, mit diesen Instrumenten umgehen zu können. Kompetenz und Durchsetzungsfähigkeit der Eltern gegenüber der öffentlichen Verwaltung sind entscheidend für die Quantität und Qualität des genehmigten Platzes. Dies führt zwangsläufig zu Benachteiligungen für die Kinder, die besonders auf Unterstützung und Förderung angewiesen sind und deren Eltern diesen Herausforderungen eher unwillig gegenüberstehen, sie nicht verstehen oder im Umgang damit überfordert sind.

- Aus der Perspektive der Eltern führt die mit der Subjektfinanzierung angestrebte Wirtschaftlichkeit dazu, dass sie mit eingeschränktem Wunsch- und Wahlrecht und sogar mit höheren Beiträgen rechnen müssen, wenn die Wohnsitzgemeinde den Platz in einer anderen Gemeinde nicht finanziert wie dies in Bayern zum Teil geschieht.

- Tendenziell sind die subjektbezogenen Systeme mit hohem Verwaltungsaufwand sowohl bei den öffentlichen wie 
den freien Trägern verbunden. Hier werden Finanzströme in Bereiche umgesteuert, die Eltern und Kinder am wenigstens zugute kommen.

- Die Refinanzierung der Personalkosten orientiert sich an der Anwesenheitszeit der Kinder, Planungszeiten, Weiterbildung und Zeiten für Elternarbeit, die erheblichen Einfluss auf die Qualität haben, werden nicht berücksichtigt.

- Das wirtschaftliche Risiko bei Nicht Belegung einzelner Plätze wird vollständig auf den Träger übertragen. Die Konsequenz sind befristete Verträge oder Teilzeitverträge für die Fachkräfte, was für den Erhalt von Qualität und Kontinuität gerade in der Arbeit mit kleinen Kindern kontraproduktiv ist. Zeitverträge und Teilzeiteinkommen befördern zudem die Entwicklung prekärer Lebenssituationen von Fachkräften, die zunehmend selber von Armut betroffen sind. Die mit der Subjektfinanzierung angestrebte Wirtschaftlichkeit und Effizienzsteigerung geht zu Lasten der Fachkräfte und der Kinder. Subjektfinanzierung erfolgreich umgesetzt würde bedeuten, die finanziellen Beschränkungen durch festgelegte Budgets aufzuheben, fachlich angemessene personelle Standards festzulegen und eine am Bedarf des Kindes orientierte Entscheidung über den zeitlichen Umfang des Angebotes einzuführen. Befürworter der Subjektfinanzierung heben die stärkere Wettbewerbs- und Marktorientierung hervor, die vor allem die Seite der Nachfragenden, der Eltern, stärken soll.

Dazu ist kritisch anzumerken, dass Markt und Wettbewerbsfaktoren zunächst ein umfassendes Angebot voraussetzen. Dies mag in einigen Ballungsgebieten für einen Kindergartenplatz erfüllt sein, im Westen trifft dies schon nicht mehr bei Ganztagsplätzen und Krippenplätzen zu. In kleinstädtischen und ländlichen Regionen ist ein Wettbewerb aufgrund der geringen Auswahl gar nicht möglich.

Wettbewerb verlangt Mobilität und gute Information der Nachfragenden auch dies kann bei Eltern nicht vorausgesetzt werden. Bisher fehlen empirische Belege dafür, dass Druck durch Wettbewerb anreizfördernde Wirkung hätte oder gar ein geeignetes Instrument zur Gewährleistung von Chancengleichheit wäre. Ein Anreiz für die Weiterentwicklung flexibler und bedarfsgerechter Angebote dürfte sich vielmehr aus einer gesicherten Finanzierung ergeben, die Träger nicht zwingt, die risikoärmste und kostengünstigste Variante von Angeboten zur Verfügung zu stellen.

\section{Resümee}

Der OECD-Bericht »Starting Strong « kam im Jahr 2001 zu der Einschätzung, Erziehung, Bildung und Betreuung werde in Deutschland häufig eher vom aktuell verfügbaren Budget bestimmt als von dem Bemühen, das Budget auf die wachsenden Herausforderungen und Qualitätsanforderungen abzustimmen. Dies gilt immer noch im Jahr 2008.

Es gibt in Deutschland erheblichen Nachholbedarf bei der Entwicklung eines leistungsgerechten Finanzierungssystems für Kindertageseinrichtungen, das den Anforderungen gerecht wird, die an den Elementarbereich gestellt werden: Ausgleich von Benachteiligungen, Prävention von Armut, individuelle Unterstützung von Bildungsbiografien.

\section{Anmerkungen}

(1) Stefan Sell, Quantitative und Qualitative Abschätzung der Einführung eines Geldleistungsgesetzes zur Finanzierung der Kindertagesbetreuung in Deutschland; ibus discussion papers Nr. 012005.

(2) Vgl. Johannes Münder u. a., Frankfurter Kommentar zum SGB VIII: Kinder- und Jugendhilfe, Weinheim 2006.

(3) $\mathrm{Zu}$ den Reformbedarfen in der Finanzierung erscheint ein Positionspapier des Diakonischen Werkes der EKD; zu beziehen per E-Mail: beneke@diakonie.de.

\section{Das neue Beraterhandbuch zum Ausländer- recht}

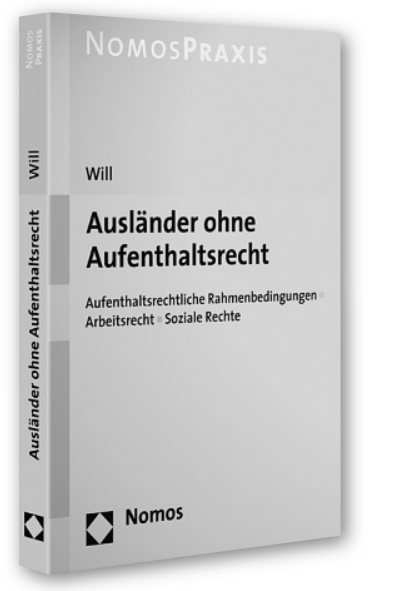

\section{Ausländer ohne \\ Aufenthaltsrecht}

Aufenthaltsrechtliche Rahmenbedingungen | Arbeitsrecht | Soziale Rechte

Von RAin Prof. Dr. Annegret Will, Ev. Fachhochschule Ludwigshafen 2008, 296 S., brosch., 39,- $€$, ISBN 978-3-8329-2500-O

Für die Beratung von Ausländern, die kein Aufenthaltsrecht (mehr) oder nur ein prekäres Aufenthaltsrecht besitzen, sind Kenntnisse des Aufenthalts-, Arbeits- und Sozialrechts ebenso unentbehrlich wie straf- und datenschutzrechtliches Wissen. Das neue Handbuch behandelt sämtliche Rechtsfragen, die sich in der Beratungsarbeit stellen.

Topaktuell: Auf neuestem Stand berücksichtigt das Werk bereits das Gesetz zur Umsetzung aufenthalts- und asylrechtlicher Richtlinien der EU/2. Änderungsgesetz.

\section{Nomos}

Bitte bestellen Sie bei Ihrer Buchhandlung oder bei Nomos | Telefon 07221/2104-37 | Fax -43 | www.nomos.de | sabine.horn@nomos.de 